to those of the template. The MIPs become capable of selectively absorbing the template species. Because of the stability, predesigned selectivity, and easy preparation of MIPs, they have been used for affinity chromatographic stationary phases, artificial antibodies, synthesis mediators, sensor components, and adsorbents for solid-phase extraction. The common forms of MIPs are bulk materials and membranes. N. Minoura of the National Institute of Advanced Industrial Science and Technology in Japan, K. Idei of Japan Science and Technology Corporation, A. Rachkov of Nihon University, and colleagues have prepared and characterized molecularly imprinted polymer membranes with a photoregulated ability to interact reversibly with a predetermined compound.

The researchers report their work in the December 16, 2003 issue of Chemistry of Materials. In their synthesis, p-phenylazoacrylanilide (PhAAAn), a derivative of azobenzene with photoresponsive properties, was selected as the functional monomer. After it was polymerized, the polymer's chemical properties changed reversibly by isomerization of this photoresponsive chromophore. Dansylamide (DA) was chosen as the template in the MIP synthesis because DA has the ability to decrease the rate of photoisomerization in solutions of PhAAAn. To improve the membrane's flexibility, the cross-linker consisted of a mixture of ethylene glycol dimethacrylate (EGDMA) and tetraethylene glycol diacrylate (TEGDA). After DA, PhAAAn, EGDMA, and TEGDA were dissolved in acetonitrile, the freeradical polymerization was initiated by 2,2-azobis (4-methoxy-2,4-dimethylvaleronitrile) between two glass plates at $35^{\circ} \mathrm{C}$. The formed MIP membrane was about $80 \mu \mathrm{m}$ thick. Another control membrane was prepared using the same procedure without adding the template DA.

The capacity and selectivity of the membranes were tested by incubating the membranes in the DA acetonitrile solutions at a concentration of $10 \mu \mathrm{M}$. The MIP membrane absorbed more DA than the control membrane, and its capacity is about $1.2 \mathrm{nmol}$ of DA $/ \mathrm{cm}^{2}$, or $0.15 \mathrm{nmol} / \mathrm{mm}^{3}$. The affinity of the binding sites can be controlled by illuminating the samples with the light of different wavelengths. Upon illumination with UV light, the absorbed DA from the MIP membrane was released. Following a second illumination with visible light, the concentration of DA in solution and in the MIP membrane returned to levels close to those seen before the application of UV radiation. The control membrane did not show this property. Because the MIP membrane was tailored by imprinting DA, it also showed the selectivity and recognition to DA rather than to other molecules.

Such MIP membranes showed a higher absorption level of DA than other reported levels of photoinduced effects in azobenzene-containing materials. The researchers said that molecular imprinting technology can be used to design photonic devices such as a photoswitches and photosensors.

YUE HU

\section{Tandem OLEDs Deliver High Luminous Efficiency}

Organic light-emitting diodes (OLEDs) are being developed for their application in displays, and therefore a high brightness is required. Extreme brightness $\left(>10^{5} \mathrm{~cd} / \mathrm{m}^{2}\right)$ using current densities as large as $1 \mathrm{~A} / \mathrm{cm}^{2}$ has been reported for OLEDs. However, at these high current densities, failure of the device due to coulombic degradation and excessive heating is a major problem. Therefore, research has focused on a way to obtain an OLED-based device with high brightness and high efficiency at low current densities. At the Display Technologies Laboratory of the Eastman Kodak Company in Rochester, N.Y., L.S. Liao, K.P. Klubeck, and C.W. Tang have designed a tandem OLED that can give luminous efficiencies as large as $136 \mathrm{~cd} / \mathrm{A}$ for current densities of $1.0 \mathrm{~mA} / \mathrm{cm}^{2}$.

In order to build a tandem OLED structure, multiple active regions are stacked vertically in a device in series. The main issue when designing such a structure is the choice of the intermediate electrode

\section{FOR MORE RESEARCII NEWS ON MATERLALS SCIENCE ...}

. access the Materials Research Society Web site:

www.mrs.org/gateway/ matl_news.html material between the units, which is the region that should not only provide connection, but also hole and electron injection, separately, into the adjacent electroluminescent (EL) units. In the past, researchers have used transparent inorganic layers such as indium tin oxide, $\mathrm{Mg}: \mathrm{Ag}$ /indium zinc oxide, or $\mathrm{V}_{2} \mathrm{O}_{5}$ as intermediate electrodes to connect the OLEDs. As an alternative, Liao and colleagues have fabricated a tandem OLED structure using an organic connecting unit to replace the inorganic intermediate electrode. According to their article scheduled for publication in the January 5 issue of Applied Physics Letters, key advantages to using the organic connecting unit include increased transparency compared with inorganic materials, excellent electrical properties, ease of fabrication by thermal evaporation, and full compatibility with OLED production processes.

The connecting units in the tandem OLEDs consist of a bilayer of doped organic thin films in contact with each other to form a doped organic $p-n$ junction at their contact interface. The $n$-type doped layer is tris(8-hydroxyquinoline) aluminum or 1,3,5-tris $(N$-phenylbenzimidazol-2-yl)benzene doped with $\mathrm{Li}$, and the $p$-type doped layer is 4,4 -bis-(1-naphthyl-N-phenylamino)-biphenyl doped with $\mathrm{FeCl}_{3}$. The research team found that the emission of one EL unit is practically independent of the emission of the other EL units in the tandem structure, and thus the units behave as if they are individual devices simply connected in a series. As an example, the researchers show the electroluminescence spectra of a tandem OLED having green and red emission. They also show the EL characteristics of fluorescent and phosphorescent devices with one, two, or three EL units. The researchers show that the luminance of a tandem OLED structure with $N$ EL units is $N$ times that of a nontandem unit when operated under the same current density. As the lifetime of an OLED is basically in inverse proportion to its operating current, the researchers said that an important advantage of the tandem structure is that the operational lifetime of the OLED device can be substantially improved. The researchers also said that this tandem OLED structure is also suitable for some special lighting applications.

ROSALIA SERNA

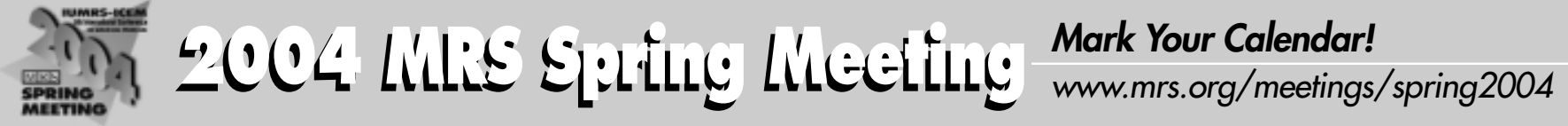

